\title{
Niveles de alfabetización en la UAA
}

MA. TERESA FERNÁNDEZ LOMELÍN Y MARGARITA CARVAJAL CIPRÉS

Departamento de Educación/UAA

\section{RESUMEN}

\begin{abstract}
A partir de la definición de los niveles de alfabetización y de su especificación en diversas categorías, así como de las prácticas de lectura y escritura de los estudiantes universitarios, se realizó esta investigación tomando como muestra a 1362 alumnos de los diferentes centros de la UAA. Los resultados son sorprendentes en tanto que la mayoría de los estudiantes apenas llega al nivel de alfabetización básica y otros muchos $(35 \%)$ se ubican dentro del analfabetismo funcional. Estos datos deben llevar a los profesores universitarios a reflexionar sobre la necesidad de trabajar en el desarrollo y perfeccionamiento de las habilidades de lectura y la escritura de los estudiantes.
\end{abstract}

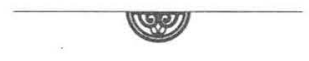

\section{ABSTRACT}

\section{LEVELS OF LITERACY IN THE UAA}

From the definition of different levels of literacy and its specification in several categories, and also by observing the practice of reading and writing of the students, this investigation was realized taking 1632 students from different centers of the UAA as a sample. The results were surprising: most of the students hardly reach the basic level of literacy, while many others (35\%) are in the functional level. This information may lead the teachers of the University to reflect about the development and mastery of the writing and reading skills among students. 


\section{INTRODUCCIÓN}

La frase que da título a este artículo puede parecer poco pertinente, sobre todo cuando se participa en el proceso de enseñanza en una institución de educación superior como lo es la Universidad Autónoma de Aguascalientes.

Si bien no es costumbre hablar de alfabetización cuando se hace referencia a una institución de educación superior, en este trabajo si lo hacemos ya que se parte del supuesto de que los alumnos continúan alfabetizándose a un nivel diferente que el básico o inicial aprendido en la educación primaria. Bajo este principio, la información que se presenta en este escrito invita a la reflexión sobre nuestra labor como docentes universitarios.

El presente artículo es producto de una investigación realizada durante 1997 y 1998 con los estudiantes de los semestres primero, quinto y décimo de 13 de las carreras ofrecidas por la UAA. El objetivo central fue la identificación de los niveles de alfabetización (entendida como dominio de la lectura y la escritura) que los estudiantes de dichas carreras alcanzaban frente a un texto expositivo de divulgación científica. Como elemento complementario, se indagó sobre algunas prácticas preuniversitarias, universitarias y familiares que pudiera suponerse, favorecen la práctica de la lectura y la escritura.

La lectura y la escritura son dos procesos que implican el desarrollo de ciertas habilidades desde los primeros años de vida y son esenciales para el desenvolvimiento social del individuo. La escritura permite la acumulación y divulgación del conocimiento reunido por muchas manos durante mucho tiempo, de tal manera que la masa de conocimientos e información a la que cualquier individuo tiene acceso, gracias a la escritura, es inmensa.

La capacidad de leer y escribir requerida para explotar y contribuir a ese acervo, se vuelve cada vez más especializada. Depende no solamente de las habilidades de cada uno, sino de un profundo 
entendimiento de los dominios representados por los textos que contienen dichos conocimientos.

Es de esperarse que los alumnos que realizan estudios universitarios tengan estas habilidades desarrolladas a tal punto que, por un lado, puedan acceder a este cúmulo de conocimientos especializados para poder preparase profesionalmente, y por otro, que fueran capaces de producir escritos coherentes, ricos en vocabulario y sin faltas de ortografía. Ambas cosas les permitirán desempeñarse en el ámbito laboral de manera exitosa.

\section{LA ALFABETIZACIÓN}

Leer y escribir suponen un aprendizaje, un esfuerzo y también una creatividad que rebasa la capacidad de entender y construir oraciones con una estructura gramatical más o menos aceptable. Los procesos de lectura y escritura, entendidos como tales desde la psicología cognitiva, constituyen lo que normalmente se conoce con el término "alfabetización". ${ }^{1}$

Para Rihes, ${ }^{2}$ la alfabetización consiste no solamente en saber, sino también en la familiarización con las habilidades lingüísticas correlativas de hablar y escuchar; de esta forma, las habilidades de leer, escribir, hablar y escuchar son instrumentos fundamentales para el aprendizaje.

La alfabetización es equiparable con la competencia en el uso de un escrito con uno o varios propósitos particulares, esto es, la familiaridad general con la naturaleza y función de un escrito es

${ }^{1}$ D. Baker y B. Street, "Literacy and Numeracy: concepts and Definitions" en: Hussein y Postlethwaite The International Encyclopedia of Education. Second ed. London, Pergamon Press. Vol. 6, 1994, pp. 3453-3459. D. R. Olson, Literacy en: Hussen y Postlethwaite The International Encyclopedia of Education. Second ed. London, Pergamon Press. Vol. 6, 1994, pp. 3443-3446

${ }^{2}$ Citado por David Hawkins, "The Roots of Literacy" en: S. Graubard, Literacy, an overview by 14 experts. New York, Rhe Noonday Press, 1991. pp. 1-14. 
conocida como alfabetización básica; mientras que la utilización de un escrito para un conjunto de funciones es conocida como alfabetización funcional. La competencia en la formación e interpretación de textos especializados con un dominio experto, es nombrada en ocasiones como alfabetización de élite.

Algunos ejemplos del nivel mínimo de utilización de las habilidades involucradas en la alfabetización son: la lectura de signos y direcciones o instrucciones simples; escribir mensajes simples necesarios para la comunicación entre los ciudadanos, como por ejemplo, el nombre. Por otro lado, un nivel de alfabetización de élite comprende habilidades para leer comprensivamente pasajes complejos o construir e interpretar un manual técnico.

Otros dos grupos a distinguir dentro de la categorización de la alfabetización que no tienen que ver con los propósitos sino con la falta de ellos, son, por un lado el analfabetismo, que indica una ausencia o poca familiaridad con la lectura y la escritura, y por otro el analfabetismo funcional, que se caracteriza por la pérdida o deterioro de las habilidades para leer y escribir por la falta de uso o la práctica poco sistemática ${ }^{3}$.

La alfabetización en la universidad involucra el aprendizaje de la cultura, aprendiendo términos que son distintivos de los rituales, valores, estilos de lenguaje y conducta. De esta manera se espera que los estudiantes en estos niveles, al menos se ubiquen en la categoría de alfabetos funcionales, si no es que de élite.

Dado que en este trabajo se considera que el nivel de alfabetización que alcanza un sujeto es producto de la interacción de dos procesos en desarrollo, el de lectura y el de la escritura, se presenta brevemente una descripción de cada uno de ellos.

\footnotetext{
${ }^{3}$ L. M. Fernández, Una aproximación a la comprensión lectora a través de sus niveles, Tesis presentada para obtener el grado de Maestría en Educación, UAA, 1996.
} 


\section{EL PROCESO DE LECTURA}

Desde la psicología cognitiva leer es sinónimo de comprender un texto, lo cual supone construir un significado a partir de la información ofrecida por un autor determinado.

De esta manera, la comprensión lectora es el proceso de elaborar el significado por la vía de aprehender las ideas relevantes del texto y relacionarlas con los conocimientos y las experiencias previas del lector, es decir, es "el proceso a través del cual el lector interactúa con el texto"."

La eficiencia lectora se concibe como la capacidad de poseer un nivel de comprensión que permita reconocer el contenido del discurso en diferentes tipos de textos, según la finalidad comunicativa y el auditorio a los que van destinados, así como la capacidad de emitir una opinión personal sobre las ideas que contiene el discurso. De esta manera, se pueden considerar distintos niveles de comprensión lectora:

A. Nivel literal: Consiste en distinguir y comprender lo que el autor quiere decir de manera directa. Se caracteriza porque el lector se concentra en lo impreso y obtiene el significado literal sin evaluar las ideas ni reflexionar sobre ellas; requiere del conocimiento de un vocabulario, un esquema referencial del lector (lecturas y conocimientos previos) y habilidades para encontrar las ideas principales, la secuencia de las mismas y la localización de la información pertinente y relevante. Barret ${ }^{5}$ se refiere a este nivel como "recuperación de la información explícitamente descrita en el texto sin añadiduras ni omisiones".

Este nivel comprende dos etapas esenciales: la determinación del tema y la localización de las ideas principales, lo que a su vez

\footnotetext{
+ David Cooper, Como mejorar la comprensión lectora. España. AprendizajeVisor, 1990.

${ }^{5}$ Citado en Aníbal Puente (coord.). Comprensión de lectura y acción docente. Madrid. Fundación Germán Sánchez Ruipérez, 1991. p. 35
} 
supone una lectura global del texto (de principio a fin sin omitir los detalles), y el conocimiento del vocabulario, es decir, el entendimiento de las palabras y expresiones que usa el autor, el conocimiento de su significado literal y, además, del sentido preciso en el que son empleadas en el texto.

- Este nivel se manifiesta cuando a una pregunta expresa sobre un texto, el sujeto responde retomando textualmente las ideas requeridas. Aquí estamos situados a un nivel relativamente bajo, ya que el sujeto limita su lectura a lo que la página impresa indica sin llegar a hacer una interpretación.

B. Nivel Reorganizativo: Consiste en organizar de otra manera el texto a través de: 1) procesos de clasificación y síntesis, el primero de los cuales ya se realizó en el nivel literal; 2) identificación de las relaciones entre las palabras y expresiones principales (aquí se necesita relacionar los significados analizando el vocabulario y las relaciones semánticas entre los términos); 3) recapitulación del contenido y revisión de la integración acumulada para seguir el desarrollo que interesa.

Los lectores no han comprendido un texto en un nivel reorganizativo si sólo repiten la información tal cual viene en el texto; alcanzan este nivel cuando han establecido conexiones lógicas entre las ideas y pueden expresarse de otra manera.

Este nivel generalmente se manifiesta cuando se solicita a un lector que elabore un resumen o síntesis de textos. Cuando realiza esta tarea dándole una organización diferente al texto, respetando las ideas principales, un lector se encuentra en un nivel reorganizativo.

C. Nivel inferencial. A este nivel el lector puede explicar, interpretar o comentar el contenido del texto o generalizar la información contenida en él a situaciones de la vida cotidiana. Para que el lector pueda interpretar un texto requiere ir analizando el significado de los conceptos generales. En esta dimensión, el objetivo es explicar o comentar el texto. 


\section{EL PROCESO DE ESCRITURA}

Muchos maestros e investigadores han resaltado la importancia de la escritura como un medio para la exploración de la información y el conocimiento en las distintas disciplinas del currículum. Flower y Hayes ${ }^{6}$ identifican a la escritura como una estrategia para la resolución de problemas que permite a las personas concretizar y explorar las representaciones no verbales. Asimismo, señalan que existe una fuerte relación entre el pensamiento y la escritura para adquirir o manifestar conocimiento. Esta habilidad para usar la escritura para solidificar y expandir los propios conocimientos, experiencias y observaciones es de crucial importancia en la vida académica y profesional.

La escritura permite al individuo descubrir lo que piensa, probablemente porque, como Emig resume, la escritura guía la integración del material, permite su revisión y su reevaluación, ayuda a las conexiones y es activada y dictada por los propios patrones de pensamiento y de acción.

Cooper y sus colaboradores, ${ }^{8}$ realizaron un estudio con 400 estudiantes de nuevo ingreso en la Universidad Estatal de Nueva York, en el cual se solicitó a los participantes que escribieran un ensayo sobre un tema específico. Uno de los hallazgos que se hicieron fue en el sentido de que los estudiantes tenían un dominio adecuado de las habilidades de orden inferior referidas al uso mecánico de cuestiones gramaticales o de vocabulario. Sin embargo, en las habilidades que implicaban análisis crítico de información con argumentos sólidos, tenían carencias.

"L. Flower y J. Hayes, "The cognition of discovery: defining a rhetorical problem" en: College Composition and Communication, I. I. 1984 pp. 120-60.

' J. Emig, "Writing as a mode of learning" en: College Composition and Communication, 28, 1977 pp. 122-8.

${ }^{8} \mathrm{C}$. Cooper et. al. "Studying the writting abilities of a university freshman class: strategies from a case study." en New Directions in Composition Research. New York, The Guilford Press, 1984, pp. 12-21. 
Por otro lado, en una investigación realizada por Radino, ${ }^{9}$ acerca de problemas de comunicación escrita, el autor menciona que los alumnos que llegan a la universidad tienen un manejo deficiente de su lengua. Todos sus escritos están plagados de errores de ortografía y de sintaxis; carecen de puntuación; su vocabulario es pobre, reiterativo e impreciso; el conjunto es confuso y a menudo incoherente. En suma, son incapaces de expresar sus ideas por escrito en forma clara y ordenada debido a que no conocen su lengua. Esto se ve reflejado en todas las materias e incide negativamente en el rendimiento académico de los alumnos.

Los errores encontrados, señala el autor, pueden explicarse como una interferencia del código oral en el código escrito. Los estudiantes manifiestan que al escribir utilizan las mismas reglas de estructuración que emplean al hablar. En otras palabras, los estudiantes no tienen clara conciencia de que la escritura no es un mero código sustitutivo del lenguaje oral, ni una simple convención de transcripción de lenguaje oral, sino que se organiza según reglas propias que obedecen a sus condiciones específicas de producción y transmisión, condiciones bastante diferentes de las de la oralidad.

El problema global radica en el desconocimiento de la especificidad del registro escrito, desconocimiento que es teórico (es decir, de comprensión) y práctico (de producción o ejercicio). Esto es así y en el proceso de escritura intervienen una multiplicidad de habilidades, pero para este estudio se seleccionaron únicamente algunos aspectos de ortografía y coherencia, observados a través de un instrumento diseñado tanto para tal fin como para identificar los niveles de comprensión lectora.

El instrumento consistió en la presentación de un texto expositivo de divulgación científica, cuyo tema remite a la preocupación del ser humano por su mejoramiento genético, haciendo alusión al proceso de

\footnotetext{
${ }^{9}$ Citado en J. Robert, Las capacidades Humanas. Un enfoque desde el procesamiento de la información. Barcelona, España, Labor Universitaria, 1998, pp. 75-106.
} 
clonación. Enseguida se formularon un conjunto de ítems abiertos a los cuales tenían que dar respuesta los estudiantes conforme a lo leído.

El análisis de dichas respuestas permitió relacionar los aspectos del proceso de lectura con el de la escritura cuyo producto final nos condujo a la identificación de niveles de alfabetización de los estudiantes de la muestra.

\section{NiveleS DE ALFABETIZACión}

Se construyeron cuatro niveles de alfabetización, al interior de los cuales se distinguieron categorías conforme a lo identificado en el instrumento diseñado para observar el nivel de comprensión lectora y los aspectos de escritura.

a) Alfabetización de élite es aquella en la cual el sujeto logra formar e interpretar textos especializados con un dominio experto. b) Alfabetización funcional es aquella en la que la escritura y la lectura se utilizan para un grupo específico de funciones.

c) Alfabetización básica permite la familiaridad general con la naturaleza y función de la lectura y la escritura.

d) Analfabetismo funcional es la pérdida o deterioro de las habilidades de leer y escribir por falta de uso o práctica poco sistemática.

La construcción de niveles y categorías de alfabetización se realizó primeramente compactando en índices lo correspondiente a ortografía y coherencia y niveles de comprensión lectora y en segundo término se consideraron conjuntamente ambos aspectos, quedando como sigue:

\section{Alfabetización de élite}

Si los estudiantes lograron un nivel inferencial de lectura, no tuvieron errores ortográficos y además cubrieron los aspectos evaluados en coherencia de manera adecuada. 
Alfabetización funcional

Categoría 1. Si lograron un nivel inferencial en lectura, tuvieron errores ortográficos, pero escribieron coherentemente.

Categoría 2. Si el nivel de comprensión lectora fue reorganizativo, tuvieron buena ortografía y coherencia aceptable en la escritura. Categoría 3. Nivel de comprensión reorganizativo, ortografía deficiente y coherencia aceptable.

Alfabetización básica.

Categoría 1. Nivel de lectura reorganizativo, buena ortografía pero coherencia deficiente.

Categoría 2. Nivel de comprensión inferencial, ortografía deficiente y coherencia mala.

Categoría 3. Nivel de comprensión reorganizativo, ortografía adecuada pero coherencia deficiente.

Categoría 4. Nivel literal de comprensión, ortografía adecuada y coherencia buena.

Categoría 5. Nivel de comprensión literal, ortografía deficiente pero coherencia satisfactoria.

Categoría 6. Nivel reorganizativo de lectura, ortografía y coherencia inadecuadas.

Analfabetismo funcional

Categoría 1. Nivel literal de lectura, ortografía satisfactoria pero coherencia inadecuada.

Categoría 2. Nivel de lectura literal, ortografía y coherencia no satisfactorias.

Categoría 3. Todas las combinaciones posibles de ortografía y coherencia, pero no se alcanza ningún nivel de comprensión lectora.

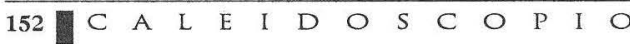


Categoría 4. No se alcanza ningún nivel y se tienen ortografía y coherencia no satisfactorias.

\section{CUADRO 1}

Niveles de alfabetización en la muestra global

\begin{tabular}{|c|c|c|c|}
\hline NIVELES & & $\mathrm{f}$ & $\%$ \\
\hline \multirow[t]{2}{*}{ Alfabetización de Élite } & & 5 & 0.4 \\
\hline & Subtotal & 5 & 0.4 \\
\hline Alfabetización Funcional 1 & & 66 & 4.8 \\
\hline Alfabetización Funcional 2 & & 29 & 2.1 \\
\hline \multirow[t]{2}{*}{ Alfabetización Funcional 3} & & 154 & 11.3 \\
\hline & Subtotal & 249 & 18.2 \\
\hline Alfabetización Básica 1 & & 2 & 0.1 \\
\hline Alfabetización Básica 2 & & 5 & 0.4 \\
\hline Alfabetización Básica 3 & & 1 & 0.1 \\
\hline Alfabetización Básica 4 & & 114 & 8.4 \\
\hline Alfabetización Básica 5 & & 516 & 37.9 \\
\hline \multirow[t]{2}{*}{ Alfabetización Básica 6} & & 16 & 1.2 \\
\hline & Subtotal & 654 & 48.1 \\
\hline Analfabetismo Funcional 1 & & 8 & 0.6 \\
\hline Analfabetismo Funcional 2 & & 46 & 3.4 \\
\hline Analfabetismo Funcional 3 & & 360 & 26.4 \\
\hline \multirow[t]{2}{*}{ Analfabetismo Funcional 4} & & 40 & 2.9 \\
\hline & Subtotal & 454 & 33.3 \\
\hline Total de la muestra & & 1362 & 100 \\
\hline
\end{tabular}

Grosso modo, la información que muestra este cuadro indica que alrededor de un $80 \%$ de alumnos de la muestra manifiesta tener un nivel de alfabetización entre el básico y el funcional, es decir, estos resultados coinciden, por un lado, con lo que menciona Cooper en lo relativo a la dificultad de los estudiantes universitarios de lograr un escrito que manifieste un análisis crítico, que correspondería en este caso al nivel inferencial de lectura, y por otro, a lo que alude Radino en relación con la carencia de una buena ortografía y/o coherencia adecuada en el texto.

$$
\begin{array}{llllllllllllll}
\text { C } & \text { A } & \text { L } & \text { E } & \text { I } & \text { D } & \text { O } & \text { S } & \text { C } & \text { O } & \text { P } & \text { I } & \text { O } & \mathbf{1 5 3}
\end{array}
$$


En toda la muestra pueden identificarse únicamente 5 sujetos que lograron un nivel de élite y 154 que leen a nivel inferencial, escriben con cierta coherencia pero cuentan con mala ortografía (categoría 3 de alfabetización funcional).

Del lado contrario, se identifican 360 alumnos en un nivel de analfabetismo funcional 3 (no alcanzan ningún nivel de comprensión lectora, aunque cuentan o con buena ortografía o coherencia adecuada en sus escritos) y un $3 \%$ que se encuentran en la última categoría de analfabetismo funcional.

El análisis de los niveles de alfabetización de acuerdo a los centros en que se ubican los estudiantes que participaron en el estudio, revela lo siguiente (recuérdese que no se aplicaron cuestionarios a todas las carreras): ${ }^{10}$

CUADRO 2

Niveles de alfabetización por Centro

\begin{tabular}{|c|c|c|c|c|c|c|c|c|c|c|c|c|}
\hline \multirow{3}{*}{ NIVELES } & \multicolumn{12}{|c|}{ CENTROS } \\
\hline & \multicolumn{2}{|c|}{$\begin{array}{l}\text { C. Ciencias } \\
\text { Agropecua. }\end{array}$} & \multicolumn{2}{|c|}{$\begin{array}{l}\text { C. Ciencias } \\
\text { Básicas }\end{array}$} & \multicolumn{2}{|c|}{$\begin{array}{l}\text { C. Ciencias } \\
\text { Biomédicas }\end{array}$} & \multicolumn{2}{|c|}{$\begin{array}{l}\text { C. Ciencias } \\
\text { del Diseño } \\
\text { y de la Const. }\end{array}$} & \multicolumn{2}{|c|}{$\begin{array}{c}\text { C. Ciencias } \\
\text { Económicas } \\
\text { y Adm. }\end{array}$} & \multicolumn{2}{|c|}{$\begin{array}{l}\text { C. Ciencias } \\
\text { Sociales y H. }\end{array}$} \\
\hline & f & $\%$ & $\mathrm{~F}$ & $\%$ & $\mathrm{~F}$ & $\%$ & $\mathrm{~F}$ & $\%$ & $f$ & $\%$ & $\mathrm{~F}$ & $\%$ \\
\hline Élite & 1 & 0.8 & 1 & 0.5 & 0 & 0 & 0 & 0 & 2 & 0.8 & 1 & 0.2 \\
\hline Función 1 & 6 & 4.7 & 8 & 3.7 & 3 & 1.9 & 7 & 5.3 & 14 & 5.5 & 28 & 5.9 \\
\hline Función 2 & 4 & 3.1 & 2 & 0.9 & 4 & 2.5 & 2 & 1.5 & 3 & 1.2 & 3 & 3 \\
\hline Función 3 & 12 & 9.3 & 21 & 9.6 & 17 & 10.8 & 11 & 8.4 & 41 & 16.2 & 11 & 11 \\
\hline Básica 1 & 1 & 0.8 & 0 & 0 & 0 & 0 & 0 & 0 & 0 & 0 & 1 & 0.2 \\
\hline Básica 2 & 0 & 0 & 2 & 0.9 & 0 & 0 & 0 & 0 & 2 & 0.8 & 1 & 0.2 \\
\hline Básica 3 & 0 & 0 & 1 & 0.5 & 0 & 0 & 0 & 0 & 0 & 0 & 0 & 0 \\
\hline Básica 4 & 14 & 10.9 & 15 & 6.8 & 16 & 10.2 & 11 & 8.4 & 19 & 7.5 & 39 & 8.2 \\
\hline Básica 5 & 30 & 23.3 & 87 & 39.7 & 63 & 40.1 & 47 & 35.9 & 106 & 41.9 & 183 & 38.7 \\
\hline Básica 6 & 3 & 2.3 & 2 & 0.9 & 2 & 1.3 & 1 & 0.8 & 3 & 1.2 & 5 & 1.1 \\
\hline Analfa. 1 & 0 & 0 & 3 & 1.4 & 2 & 1.3 & 0 & 0 & 2 & 0.8 & 1 & 0.2 \\
\hline Analfa. 2 & 5 & 3.9 & 8 & 3.7 & 8 & 5.1 & 3 & 2.3 & 5 & 2.0 & 17 & 3.6 \\
\hline Analfa. 3 & 43 & 33.3 & 62 & 28.3 & 40 & 25.5 & 43 & 32.8 & 49 & 19.4 & 123 & 26 \\
\hline Analfa. 4 & 10 & 7.8 & 7 & 3.2 & 2 & 1.3 & 6 & 4.6 & 7 & 2.8 & 8 & 1.7 \\
\hline TOTAL & 129 & & 219 & & 157 & & 131 & & 253 & & 473 & \\
\hline
\end{tabular}


Los datos anteriores, muestran una consistencia con la tendencia general presentada en el cuadro 1 . De manera general, los mayores porcentajes en todos los Centros se concentran en el nivel de alfabetización básico, específicamente categoría cinco, es decir, los alumnos muestran un nivel de comprensión literal, coherencia satisfactoria, pero ortografía deficiente.

Cabe destacar que, a excepción de los centros de Ciencias Biomédicas y de Ciencias del Diseño y la Construcción, todos los demás tuvieron por lo menos, un alumno con nivel de alfabetización de élite, es decir, logran un nivel inferencial de lectura y tienen ortografía y coherencia satisfactorias.

Otro aspecto a resaltar es que, dentro del nivel de alfabetización funcional, la categoría tres es la que presenta porcentajes más elevados con respecto a las otras dos, es decir, que los alumnos logran un nivel de comprensión reorganizativo y sus escritos son coherentes, pero su ortografía no es la deseada.

Dentro del analfabetismo funcional, hay que mencionar que los porcentajes de alumnos que muestran este nivel en los Centros de Ciencias Agropecuarias y del Diseño y Construcción, son más elevados en relación al resto de los centros, específicamente en la categoría 3 , en donde no alcanzan ningún nivel de lectura y en ocasiones tienen o buena ortografía o buena coherencia en sus escritos, pero no ambas simultáneamente.

Aunado a la identificación de niveles de alfabetización, se obtuvieron datos sobre algunos aspectos relacionados con actividades cotidianas de lectura y de escritura de los alumnos o de sus maestros y familiares durante su trayectoria escolar y su vida extraescolar.

De los cinco sujetos que se ubicaron en el nivel de alfabetización de élite, dos se sitúan en prácticas preuniversitarias (secundaria y

10 La información sobre cada una de las carreras con las cuales se trabajó puede verse en el reporte completo de esta investigación. 
bachillerato) y universitarias buenas (0.1\% del total de la muestra) y tres en prácticas regulares, manteniéndose esta tendencia en las familiares; destaca el hecho de que ninguno de los cinco se sitúa en la categoría de malas prácticas, pero tampoco en la de muy buenas.

En relación al nivel de alfabetización funcional, 189 (76\%) de $\operatorname{los} 249$ alumnos que se ubicaron en este nivel, muestran haber tenido prácticas preuniversitarias entre regulares y deficientes, al mismo tiempo que casi el 100\% (236 de 249 estudiantes) se situaron en estas mismas categorías en lo que se refiere a las prácticas universitarias.

Por lo que toca a la alfabetización básica, de 654 sujetos que se ubican en este nivel, 233 se caracterizan por tener prácticas preuniversitarias regulares y 273 en malas; situación similar se refleja en relación a las prácticas universitarias, aunque es más severo, pues en este caso son 284 y 335 alumnos ubicados respectivamente en las categorías mencionadas.

Si bien en lo relativo a las prácticas familiares un gran porcentaje se ubica en las dimensiones de regulares y deficientes (56.4 y 15.3), resalta el hecho de que 185 alumnos (28\%) se ubica en las correspondientes a muy buenas y buenas y $369(56.4 \%)$ en regulares. Este dato contrasta con los dos anteriores, indicando que posiblemente en el hogar no se fomenta el desarrollo de las habilidades relacionadas con los procesos de lectura y escritura, dado que se está hablando de un nivel básico de alfabetización.

Por último, en lo correspondiente al nivel de analfabetismo funcional, alrededor de un $80 \%$ de los estudiantes se ubica en las categorías de regulares a malas prácticas preuniversitarias, mientras que 217 alumnos de 454 muestran tener regulares prácticas universitarias y 203 deficientes.

Vuelve a llamar la atención la información obtenida en lo que corresponde a prácticas familiares, en donde se encuentran porcentajes relativamente más altos en los rubros de muy buenas y buenas prácticas familiares en relación con las preuniversitarias y univer- 
sitarias, pero nuevamente, no debe perderse de vista que se está hablando de un nivel de analfabetismo funcional, lo cual, entonces, no habla de unas muy buenas prácticas de este tipo.

Dada la información anterior y pensando que un gran porcentaje del tiempo de la mayoría de los sujetos se invierte en la escuela, la pregunta es: ¿qué pasaría si en el sistema escolar por el cual pasaron los sujetos de la muestra, incluyendo el universitario, se pusiera atención al desarrollo constante de las habilidades correspondientes a la lectura y la escritura? ¿Ascendería la cantidad de sujetos en los niveles más altos de alfabetización?

Para tener un poco más de certeza en las aseveraciones y preguntas anteriores, se realizó un análisis entre las diversas prácticas relacionadas con la lectura y la escritura y los niveles de alfabetización.

En lo relativo a las prácticas efectuadas durante la secundaria y la preparatoria, el análisis indica que la relación que tienen éstas con los niveles de alfabetización que se alcanzan es significativa, pero no más que la manifestada en prácticas universitarias.

Si se considera entonces esta información podría aventurarse la aseveración de que las prácticas que se realizan a nivel universitario tienen cierta incidencia directamente proporcional en los niveles de alfabetización logrados por los alumnos, es decir, a menores prácticas relacionadas con la lectura y la escritura en la universidad, menores son los niveles de alfabetización logrados. ${ }^{11}$

\section{ConcLusiones}

Considerando la muestra del estudio que se reporta, en general el nivel de alfabetización logrado por los alumnos es el básico, en espe-

"Para dar una idea de esta situación, del total de la muestra dentro de lo que se refiere a prácticas universitarias, $46 \%$ se coloca en la dimensión de prácticas regulares y $47.9 \%$ en deficientes, casi por mitad. Mientras en lo correspondiente a las preuniversitarias se tiene 39.1 y $38.1 \%$ respectivamente. 
cífico en lo que corresponde a la categoría 5, definida como aquella en la que los estudiantes logran un nivel de comprensión lectora literal, escriben con coherencia, pero su ortografía no es adecuada.

Otros hallazgos destacables son lo que se refieren al analfabetismo funcional, en específico los correspondientes a una ausencia de comprensión lectora y a deficiencias en la escritura, ya sea en la ortografía o en la coherencia (entre 35 y $50 \%$ de la muestra).

Estos datos invitan a la reflexión acerca de qué es lo que se hace con los alumnos en lo relativo a los procesos de lectura y escritura en su tránsito por el sistema educativo mexicano.

Se supone que cuando un alumno finaliza el $4^{\circ}$ grado de primaria es un sujeto alfabetizado por lo menos en un nivel básico, y que conforme va avanzando en escolaridad y en madurez va desarrollando cada vez más las habilidades de lectura y escritura hasta llegar a un dominio experto, es decir, a un nivel de alfabetización de élite.

Sin embargo, lo que se encuentra es que la mayoría de los alumnos, al llegar a la universidad, independientemente del semestre que cursen, no han pasado del nivel adquirido en la primaria e incluso han retrocedido.

Habrá quien piense que en niveles de educación superiores, los maestros no deben ocuparse de estas tareas; sin embargo, cabe preguntarse: ¿qué tipo de actividades de aprendizaje se instrumentan? ¿Qué exigencia se pone en ellas? Cuando se deja leer al alumno, ¿qué se le pide que haga con lo que lee? Cuando se le pide que escriba, ¿qué se le pide que redacte y con qué criterios? ¿Cuáles son las correcciones que se realizan a esos escritos? ¿Únicamente de ortografía?

Estas preguntas se enmarcan en la información obtenida de la relación entre diversas prácticas relacionadas con la lectura y la escritura y los niveles de alfabetización. La única que resultó significativa a un nivel alpha de .05 fue la relativa a las prácticas universitarias y los niveles de alfabetización. Esto no quiere decir que se deje la tarea a este nivel de educación; se llama la atención al 
respecto, ya que de quienes se está hablando es precisamente de alumnos universitarios.

En síntesis, no se pide que se dedique una clase a nivel universitario a enseñar a leer y a escribir, sino que se trabaje en la utilización y perfeccionamiento de esas habilidades en cuestiones propias de cada materia, de tal manera que los estudiantes por lo menos alcancen un nivel de alfabetización funcional.

No deja de ser paradójico, aunque en cierta forma también es ejemplar, que un siglo que se ha caracterizado por la proliferación de medios de comunicación parezca ser tan incapaz de leer en la pregunta: ¿sabe usted leer y escribir? una complejidad que rebasa a su vez la complejidad de la educación escolarizada. 
